\title{
OSCILLATING TWO-STREAM INSTABILITY AT THE RESONANCE OF OBLIQUELY INCIDENT RADIATION IN INHOMOGENEOUS PLASMAS
}

BY

$Y, Y, K U O, C$. OBERMAN, C. S. LIU AND F, TROYON

\section{PLASMA PHYSICS LABORATORY}
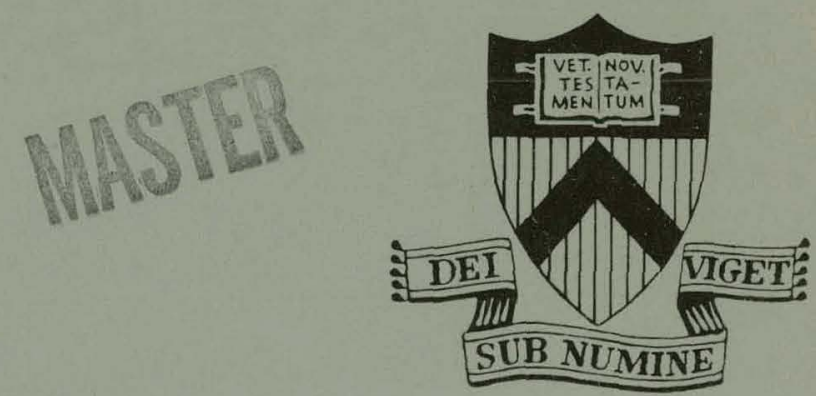

DISTRIBUTION OF THIS DOCUMENT IS UNLIMITED

\section{PRINCETON UNIVERSITY PRINCETON, NEW JERSEY}

This work was supported by U. S. Energy Research and Development Administration Contract E(11-1)-3073. Reproduction, translation, publication, use and disposal, in whole or in part, by or for the United States Government is permitted. 


\section{DISCLAIMER}

This report was prepared as an account of work sponsored by an agency of the United States Government. Neither the United States Government nor any agency Thereof, nor any of their employees, makes any warranty, express or implied, or assumes any legal liability or responsibility for the accuracy, completeness, or usefulness of any information, apparatus, product, or process disclosed, or represents that its use would not infringe privately owned rights. Reference herein to any specific commercial product, process, or service by trade name, trademark, manufacturer, or otherwise does not necessarily constitute or imply its endorsement, recommendation, or favoring by the United States Government or any agency thereof. The views and opinions of authors expressed herein do not necessarily state or reflect those of the United States Government or any agency thereof. 


\section{DISCLAIMER}

Portions of this document may be illegible in electronic image products. Images are produced from the best available original document. 


\section{NOTICE}

This report was prepared as an account of work sponsored by the United States Government. Neither the United States nor the United States Energy Research and Development Administration, nor any of their employees, nor any of their contractors, subcontractors, or their employees, makes any warranty, express or implied, or assumes any legal liability or responsibility for the accuracy, completeness or usefulness of any information, apparatus, product or process disclosed, or represents that its use would not infringe privately owned rights.

Printed in the United States of America.

Available from

National Technical Information Service

U. S. Department of Commerce 5285 Port Royal Road

Springfield, Virginia 22151

Price: Printed Copy $\$$ *; Microfiche $\$ 1.45$

*Pages

$$
1-50
$$

$51-150$

$151-325$

$326-500$

501-1000
NTIS

Selling Price

$\$ 4.00$

5.45

7.60

10.60

13.60 
Oscillating Two-Stream Instability at the Resonance of Obliquely. Incident Radiation in Inhomogeneous Plasmas

$$
\text { Y. Y. Kuo and C. Oberman }
$$

Plasma Physics Laboratory, Princeton University

$$
\text { Princeton, New Jersey } 08540
$$

and

C. S. Liu

Department of Physics and Astronomy

University of Maryland, College Park, Maryland

and

F. Troyon

Centre de Recherches en Physique des Plasmas

21 Avenue des Bains, 1007 Lausanne, Switzerland

\section{ABSTRACT}

We hiave calculated the growth rate and the threshold value for the oscillating two-stream instability for an electromagnetic wave obliquely incident on an inhomogeneous plasma. The localization of the instability is found to be in the overdense region near the threshold and to shift toward the local plasma resonance when the pump intensity increases.

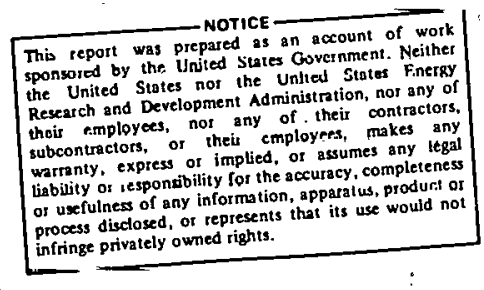




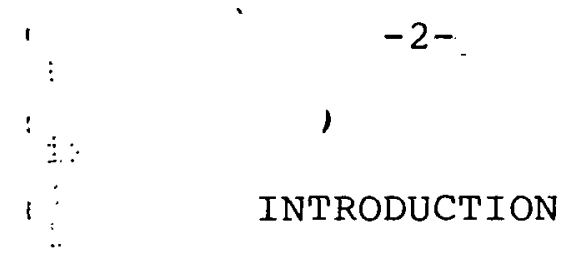

For an electromagnetic wave incident obliquely onto an inhomogeneous plasma slab, its electric field vector may have a finite component along the density gradient, causing a density oscillation at the wave frequency. At the singular point where the local. plasma frequency equals to the incident wave frequency, this electric field component resonantly drives the plasma oscillation. ${ }^{1}$ This conversion of electromagnetic wave into electrostatic wave constitutes an imporlant absorption mechanism for this obliquely incident wave. When the pump is sufficiently strong so that the driven plasma oscillation builds up to sufficiently large amplitude, it becomes unstable to parametric decay processes. The decay products are other Langmuir waves or their modified forms and the low-frequency ion density fluctuation. The ion density fluctuation in turn may change the density profile. Recent expcriments ${ }^{2}$ have indeed observed this density-profile modification near the singular puint with the subsequent generation of energetic ions and the steepening of the profile. The following theoretical questions naturally arise: (i) What is the critical amplitude of the ariven plasma oscillation at which the linear wave conversion ends and the non- linear parametric process begins; i.e., the threshold for the parametric instability? (ii) What is the growth rate of the parametric instability with the linearly transformed wave as a pump? (iii) What is the width of the unstable region compared with the width of the resonance, the former determining the region where the density profile modification takes place? and finally, (iv) what is the nonlinear 
consequence of the parametric instability of the resonantly driven plasma wave (resonance).

The first three questions can be answered by the linear analysis of the parametric process of the resonance, which yields the threshold power, the growth rate, and the width of localization of the instability. This analysis must take into account the following properties intrinsic to the resonance: (1) the locally linearly increasing (nonuniform) plasma density, (2) the structure of the pump at the resonant frequency which may be determined either by dissipation or convection. The inhomogeneity of the plasma limits the region over which the three-wave resonance condition can be met and the localization of the pump further limits the spatial extent over which the energy is supplied to the decay products. Thus, both tend to spatially confine the parametric process and significantly affect the threshold by limiting the region of interaction.

The decay of the resonance into the electron plasma wave and the ion wave has been previously calculated analytically. ${ }^{3}$. Recent numerical solution ${ }^{4}$ confirms the importance of this instability in the parameter regime for experiments of laser-induced fusion. Because the frequency of the resonance (pump) is the local plasma frequency, which is below the local Langmuir frequency one expects that the oscillating two-stream instability may also be important. In this paper, we calculate the growth rate, the threshold, and the width of localization of the oscillating two-stream instability of the resonance by including the anti-stokes component of the high-frequency mode. 


\section{$\because: \quad$ BASIC EQUATIONS}

Assume a pump field of frequency $\omega_{0}$ propagates obliquely into a slab plasma which has a linear density gradient in the $\hat{x}$-direction with scale length $L$. At the critical point where $\omega_{0}$ equals the local piasma frequency $\omega_{p}$, the $\hat{x}$ component of the pump field is resonantly converted to a localized electrostatic wave, which can be expressed as ${ }^{1}$

$$
\mathrm{E}_{\mathrm{P}}=\mathrm{E}_{\mathrm{O}}(x) \exp \left(i \omega_{0} t\right)+c \cdot c
$$

with $\mathrm{E}_{\mathrm{O}}$ given by :

$$
E_{O}(x)=\frac{\ddot{E_{0}}}{x+i \Delta}
$$

where $\tilde{\mathrm{E}}_{O}$ is the peak amplitude of the wave, and $\Delta$ is the spatial width of the wave, which is the larger value of either $L\left(\Gamma_{e} / \omega_{p e}\right)$ or $\left(I \lambda_{D}^{2}\right)^{1 / 3}$. Here, $\Gamma_{e}$ is the electron collisional frequency and $\lambda_{D}$ the electron Debye length. The electrons and ions in the plasma follow the equation of motion

$\frac{\partial \vec{v}_{s}}{\partial t}+\left(\vec{v}_{s} \cdot \vec{v}\right) \vec{v}_{s}+i_{s_{s}} \vec{v}_{s}=-\frac{\vec{\nabla}\left(\gamma_{s} N_{s} T_{s}\right)}{N_{s} m_{s}}+\frac{q_{s}}{m_{s}} \vec{E} \quad(s=e, j)$

where $\Gamma_{\mathbf{s}}$ stands for the damping rate, $\gamma_{\mathbf{s}}$ the specific heat constant, $T_{S}$ the temperature in energy units, $q_{S}$ the charge, and $m_{s}$ the mass of each species. The particle density $\mathrm{N}_{\mathbf{s}}$ and the electric field $\vec{E}$ are given by 


$$
\begin{gathered}
N_{s}=n_{o}\left(1+\frac{x}{L}\right)+n_{s}^{\prime} \\
\vec{E}=\vec{E}_{p}+\vec{E}^{\prime}
\end{gathered}
$$

with $\mathrm{E}^{\prime}$ denoting the perturbed field, $\mathrm{n}_{\mathrm{O}}$ the background density determined by $\left(4 \pi n_{0} e^{2}\right) / m=\omega_{0}^{2}$ and $n_{s}^{\prime}$ the perturbed density. 'The velocity of the electrons can also be separated into two parts, the zeroth order component driven by the pump and the perturbed part by the instabilities.

$$
\vec{v}_{e}=\vec{v}_{p}+\vec{v}^{\prime}
$$

where

$$
v_{p}=\frac{e E p}{i m_{e} \omega_{\rho}}
$$

If we combine Eq. (2) with the continuity equation and Poisson's e'quation, we obtain

$$
\begin{aligned}
& \frac{\partial^{2} n_{e}^{h}}{\partial t^{2}}+\Gamma_{e} \frac{\partial n_{e}^{h}}{\partial t}-\gamma_{e} v_{e}^{2} \nabla^{2} n_{e}^{h}+\omega_{p e}^{2}\left(1+\frac{x}{L}\right)=\frac{e}{m_{e}} \vec{\nabla} \cdot\left(n_{i}^{l} \vec{E}_{p}\right) \\
& \frac{\partial^{2} n_{i}^{l}}{\partial t^{2}}+\Gamma \frac{\partial n_{i}^{l}}{\partial t}-\gamma_{i} c_{s}^{2} \nabla^{2} \cdot n_{i}^{l}=\frac{e}{m_{i}} \vec{\nabla} \cdot\left(n_{e}^{h_{\vec{E}}}\right)
\end{aligned}
$$

where $\mathrm{h}$ and $\ell$ denote the high and low frequency components 
respectively, the quantities $\Gamma, \mathrm{c}_{\dot{s}}^{2}$, and $\mathrm{v}_{\mathrm{e}}^{2}$ are given by

$$
\begin{aligned}
& \therefore \Gamma=\Gamma_{i}+\left(m_{e} / m_{i}\right) F_{e} \\
& c_{s}^{2}=\left[T_{i}+\left(\gamma_{e} / \gamma_{i}\right) T_{e}\right] / m_{i} \\
& v_{s}^{2}=T_{e} / m_{e}
\end{aligned}
$$

Since we consider only the first upper and lower sidebands of $\mathrm{n}_{\mathrm{e}}^{\mathrm{h}}$, we write

$$
\begin{aligned}
& \left.n_{e}^{h}=\sum_{p= \pm 1} b_{p}(x) \exp \left[-i(\omega) p \omega_{0}\right) t\right]+c: c . \\
& n_{i}^{l}=a(\hat{x}) \text { exp }(-i \omega t)+c: c .
\end{aligned}
$$

which when inserted in Eqs. (6) ạn (7) yields the following coupled equations

$$
\begin{aligned}
& -\left(\omega-\omega_{0}\right)^{2} b_{1}-i \Gamma_{e}\left(\omega-\omega_{0}\right) b_{1}-\gamma_{e} v_{e}^{2} \vec{\nabla}^{2} b_{1}+\omega_{p e}^{2}\left(1+\frac{x}{L}\right) b_{1} \\
& =\frac{e}{m_{e}} \vec{\nabla} \cdot\left(\vec{E}_{O} d\right) \\
& -\left(\omega+\omega_{0}\right)^{2} b_{-1}-i \Gamma_{e}^{\left(\omega+\omega_{0}\right) b_{-1}}-\gamma_{e} v^{v^{2} \vec{\nabla}^{2} b_{-1}}+\omega_{p e}^{2}\left(1+\frac{x}{L}\right) b_{-1} \\
& =\frac{e}{m_{e}} \nabla \cdot\left(\vec{E}_{0}^{*} a\right)
\end{aligned}
$$

$-\left(\omega^{2}+i \omega \Gamma\right) a-\gamma_{i} \dot{C}_{s^{2}}^{2} \vec{\nabla}^{2} a=\frac{\dot{e}}{m_{i}} \nabla \cdot\left(\vec{E}_{0} b_{-1}+\vec{E}_{o}^{*} b_{1}\right)$ 
In order to make progress in obtaining analytical solutions for the above equations, we proceed in the WKB approximation by assuming $b_{ \pm}$equals $\exp (i k x) \hat{b}_{ \pm 1}$, and a equals $\exp (i k x) \hat{a}$ (with $k$ greater than the quantities $1 / \mathrm{L}, 1 / \Delta,\left(\partial \hat{\mathrm{b}}_{1} / \partial \mathrm{x}\right) / \hat{\mathrm{b}}$, and $(\partial \hat{a} / \partial x) / \hat{a})$. The present analysis therefore complements the numerical solution of Ref. 4 and is valid in the case of gentle gradient for which $\Delta / \lambda_{D}$ is much greater than 1 and $k_{D}$ is less than 1, so that the plasma wave is not heavily Landau damped. The resulting differential equation is

$$
\begin{gathered}
\frac{\partial^{2}}{\partial x^{2}}-2\left(\beta_{1} x-i \lambda|f|^{2}+\frac{f^{\prime}}{f}\right) \frac{\partial}{\partial x} \\
+\left[-2 \frac{f^{\prime}}{f}\left(-\beta_{1} x+\beta+i \lambda|f|^{2}\right)-\beta_{1}+i \lambda\left(|f|^{2}\right)^{\prime}+\beta_{1}^{2} x^{2}\right. \\
\left.-\beta^{2}-2 i \lambda|f|^{2} \beta_{1} x\right] \hat{b}_{-1}=0
\end{gathered}
$$

where

$$
\begin{aligned}
f(x) & =\frac{1}{\frac{x}{\Delta}-i} \\
\beta_{1} & =\frac{\omega_{p e}^{2}}{2 i k \gamma_{e} v_{e}^{2}}, \\
\beta & =+\frac{{ }^{\prime \prime \prime}\left(2 \omega+i \Gamma_{e}\right)}{2 i k \gamma_{e} v_{e}^{2}}
\end{aligned}
$$




$$
\begin{aligned}
\lambda & =\frac{-k v_{o}^{2} \omega_{o}^{2}}{2 m_{i} \gamma_{e} v_{e}^{2}\left(\omega^{2}+i \Gamma \omega-\gamma_{i} c_{s}^{2} k^{2}\right)}, \\
v_{0} & =\frac{e \hat{o}}{i m_{e} \omega_{o} \Delta}
\end{aligned}
$$

Here, $f^{\prime}$ is $d f / d x$ and $\left(|f|^{2}\right)^{\prime}$ is $d\left(|f|^{2}\right) / d x$. Under the transformation

$$
\hat{\mathrm{b}}_{1}=\xi \exp \left[+\int\left(\beta_{1} \mathrm{x}-\dot{i} \lambda|\mathrm{f}|^{2}+\frac{\mathrm{f}^{\prime}}{\mathrm{f}}\right) \mathrm{dx}\right]
$$

the differential equation can be simplified to

$$
\left(\frac{\partial^{2}}{\partial x^{2}}+F(x)\right) \xi=0
$$

where

$$
F(x)=\frac{\lambda^{2}}{\left[\left(\frac{x}{\Delta}\right)^{2}+1\right]^{2}}-\beta^{2}+\frac{2 \beta}{x-i \Delta}
$$

which is the familiar tunneling equation. The turning points of the potential well determine the width of the localization of the instabilities, : while the eigenvalues give us their threshold and growth rate. 
We consider the following two cases: (a) When the instabilities are localized within the pump width $\Delta$ so that the turning points $x_{T}$ are less than $\Delta$, and (b) when $x_{T}$ is greater than $\Delta^{\prime}$.

Case (a):

When $x_{T} / \Delta$ is less than $1, E q$. (17a) may be further simplified into

$$
\left[\frac{\partial^{2}}{\partial x^{\prime}}-\left(2 \frac{\lambda^{2}}{\Delta^{2}} x^{\prime 2}+\beta^{2}-\lambda^{2}-\frac{\beta^{2}}{2 \lambda^{2} \Delta^{2}}+\frac{2 \beta}{i \Delta}\right)\right] \xi=0,
$$

where

$$
\mathrm{x}^{\prime}=\mathrm{x}-\beta^{2} / 2 \lambda^{2}
$$

This is the familiar Weber's equation. The eigenfunction well behaved at large $x$, are

$$
\xi\left(x^{\prime}\right) \sim \exp \left(-x^{\prime 2} / x_{T}^{\prime}\right) H_{N}\left(x^{\prime} / x_{T}^{\prime}\right)
$$

where $\mathrm{H}_{\mathrm{N}}$ are the Hermite polynomials, and the eigenvalue is found from

$-(2 n+1)\left(\frac{2 \lambda^{2}}{\Delta^{2}}\right)^{1 / 2}=\beta^{2}-\lambda^{2}-\frac{\beta^{2}}{2 \lambda^{2} \Delta^{2}}+\frac{2 \beta}{i \Delta} \quad n=0,1,2, \ldots$

The turning points are at

$$
\mathrm{x}_{\mathrm{T}}^{\prime}= \pm\left(\frac{\Delta^{2}}{2 \lambda^{2}}\right)^{1 / 4}
$$


Thus, consistency with the assumption that $x_{T}$ is less than $\Delta$ requires

$$
|\lambda \Delta|>1
$$

which is satisfied at the threshold given by (26): Equation (20) can be solved for $\beta$ to yield

$\beta=\frac{1}{1-\frac{1}{2 \lambda^{2} \Delta^{2}}}\left(\frac{+i}{\Delta} \pm \frac{1}{\Delta}\left\{\left[2(\lambda \Delta)^{3}-2 \sqrt{2}(\lambda \Delta)^{2}-3(\Delta \lambda)+\sqrt{2}\right] / 2 \lambda \cdot \Delta\right\}^{1 / 2}\right)$.

Near the threshold, when $\omega$ is much less than $c_{s}{ }^{k}$, we may neglect $\omega^{2}+i \omega P_{e}$ in comparison with $c_{s}^{2} k^{2}$ in Eq. ( $15 d$ ) and $\lambda$ is real. If we let

$$
\omega=w_{r}+\dot{i}_{1} \cdot \dot{ }
$$

then from Eqs. (22) and (23) we obtain

$$
\left\{\begin{array}{l}
\omega_{r} \sim-\frac{3 k \lambda_{D}^{2}}{\Delta} \omega_{p e} \\
\omega_{i} \sim\left(\frac{v_{0}^{2}}{v_{e}^{2}}-\frac{3 \sqrt{2} k \lambda_{\mathrm{D}}^{2}}{\Delta}-\frac{\Gamma \mathrm{e}}{\omega_{\mathrm{pe}}}\right) \frac{{ }_{\mathrm{pe}}}{2}
\end{array}\right.
$$


We observe that in the inhomogeneous situation there is now a real part to the frequency, and the growth rate is reduced from the value in a homogeneous plasma by the convective loss of the plasma wave out of the pump region. The threshold condition is then

$$
\frac{\mathrm{v}_{\mathrm{th}}^{2}}{\mathrm{v}_{\mathrm{e}}^{2}} \sim \frac{\Gamma_{\mathrm{e}}}{\omega_{\mathrm{pe}}}+\frac{3 \sqrt{2} \mathrm{k} \lambda_{\mathrm{D}}^{2}}{\Delta}
$$

and the width between the turning points is

$$
D \sim 2\left|x_{T}^{\prime}\right| \sim 2\left(3 \sqrt{2} k \Delta \frac{V_{e}}{v_{o}}\right)^{1 / 2} \lambda_{D}
$$

We now consider the case of high pump power and take $\omega \gg c_{s} k$. The frequency and the growth rate are found as

$$
\left\{\begin{array}{l}
\omega_{r} \sim-\frac{3 k \lambda_{D}^{2}}{\Delta} \omega_{p e} \\
\omega_{i} \sim\left(\frac{k^{2} \lambda_{D}^{2} v_{o}^{2} m_{e}}{2 v_{e}^{2} m_{i}}\right)^{1 / 3} \omega_{p e}
\end{array}\right.
$$

which can satisfy both $\omega>c_{\mathbf{s}}{ }^{k}$ and $|\lambda \Delta|<1$ only if

$$
\frac{v_{o}^{2}}{v_{e}^{2}} \gg\left(\frac{m_{e}}{m_{i}}\right)^{1 / 2} k \lambda_{D},
$$


and

$$
\left(\frac{m_{e}}{m_{i}}\right)^{1 / 2} \frac{\Delta}{\lambda_{D}}>1 \text {. }
$$

Note that the growth rate is proportional to $\mathrm{k}^{2 / 3}$. Therefore, short-wavelength modes have stronger growth so that our WKB approximation is justified. The Landau damping, of course, limits the maximum wave number to $k_{n} \lambda_{n} 1$. Substituting Eq. (28) into (21), we find the width of the localization of the waves

$$
\mathrm{D} \sim 2\left|\mathrm{x}_{\mathrm{T}}^{\prime}\right| \sim 4\left(\lambda_{\mathrm{D}} \Delta\right)^{1 / 2}\left(\frac{\left.\mathrm{m}_{\mathrm{i}} \mathrm{v}_{\mathrm{e}}^{2} \mathrm{k} \lambda_{\mathrm{D}}\right)^{1 / 6}}{\mathrm{~m}_{\mathrm{e}} \mathrm{V}_{\mathrm{o}}^{2}}\right.
$$

Our results for $\mathrm{x}_{\mathrm{T}} / \Delta$ being less than 1 can be easily reduced to the case for a homogeneous pump by letting $\Delta \rightarrow_{. .}$. We find $\omega_{r} \rightarrow 0$ and the threshold, as well as the growth rate agree with the well-known results for the oscillating two-stream instability. 5

Case (b):

When $x_{T} / \Delta$ is larger than 1 , we need to find the roots of $F(x)=0$ in various parameter rangesi. Again, we examine the case near the threshold, when $\omega$ is much less than $c_{s}{ }^{k}$. The general situation is quite intractabile analytically. However, in the physically interesting case when $|\beta \Delta|$ is less than 1 and 
$|\lambda \Delta|$ is greater than and of the order of 1 , the turning points 6 are found as shown in Fig. i. The eigenvalue is determined, from

$\left(\exp \left\{2 i \int_{x_{2}}^{x_{4}}[F(x)]^{1 / 2} d x\right\}+1\right) \exp \left\{2 i \int_{x_{1}}^{x_{2}}[F(x)]^{1 / 2} d x\right\}=-1$,

which is shown in Appendix A, to reduce to

$$
6-\frac{3}{2^{1 / 3}}\left(\lambda^{2} \beta^{2} \Delta^{4}\right)^{1 / 3}-i \cdot \frac{8}{3} \beta \Delta=0 .
$$

, We find that

$$
\omega_{r} \sim \omega_{p e}\left(-5 \frac{k \lambda_{D}^{2}}{\Delta}-10^{-2} \frac{\Delta v_{o}^{4}}{k \lambda_{D}^{2} v_{e}^{4}}\right)
$$

and

$$
\omega_{i} \sim \omega_{p e}\left[\frac{1}{3}\left(\frac{\Delta}{6 k \lambda_{D}^{2}}\right)^{1 / 3}\left(\frac{v_{o}^{2}}{v_{e}^{2}}\right)^{4 / 3}-4 \frac{k \lambda_{D}^{2}}{\Delta}\right]-\frac{\Gamma_{e}}{2}
$$

The growth rate of the instabilities go up as $\left(\dot{v}_{0}^{2}\right)^{4 / 3}$ and $\Delta^{1 / 3}$. However, the result is true only when $|\lambda \Delta| \geq 0(1)$. If we take

$$
\lambda \Delta \sim \frac{\mathrm{v}_{\mathrm{o}}^{2} \Delta}{6 \mathrm{v}_{\mathrm{C}}^{2} \mathrm{k} \lambda_{\mathrm{D}}^{2}} \sim 5
$$

The growth rate then is 


$$
\omega_{i} \sim \omega_{p e}\left(\frac{5^{1 / 3}}{3} \frac{v_{o}^{2}}{v_{e}^{2}}-4 \frac{k \lambda_{D}^{2}}{\Delta}\right)-\frac{\Gamma_{e}}{2},
$$

and the threshold value is

$$
\frac{\mathrm{v}_{\mathrm{th}}^{2}}{\mathrm{v}_{\mathrm{c}}^{2}} \sim \frac{\Gamma_{\mathrm{e}}}{\omega_{\mathrm{pe}}}+10 \frac{\mathrm{k} \lambda_{\mathrm{D}}^{2}}{\Delta}
$$

It is worth noting that although the eigenequation (31) includes the calculation for three turning points, the contribution from the first term is negligible. Thus, the instabilities are mainly confined between $x_{1}$ and $x_{2}$, which are outside the pump width in the overdense side. This prediction is intuitively correct, since in a homogeneous pump field, the OTSI is always excited in the overdense region. The width of the localization is

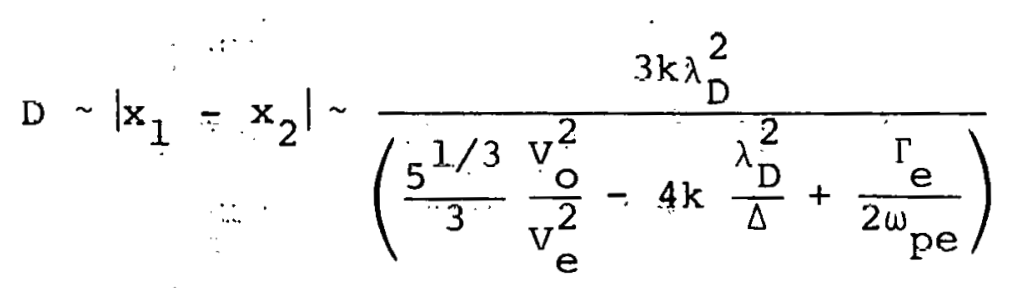

At high pump power, we shall exhibit the solution for the interesting case when both $|\beta \Delta|$ and $\left|\frac{\lambda}{\beta}\right|$ are greater than 1 . The turning points have the feature as shown in Fig. 2, and the eigenequation is found to be 


$$
\int_{x_{i}}^{x_{2}}[F(x)]^{1 / 2} d x=\left(n+\frac{1}{2}\right) \pi
$$

The evaluation of the integral is shown in Appendix $\mathrm{B}$, and we obtain

$$
\left(\lambda \beta \Delta^{2}\right)^{1 / 2} \sim \frac{\left[\Gamma\left(\frac{1}{4}\right)\right]^{2}}{8(2 \pi)^{1 / 2}}
$$

which yields

$$
\omega \sim i \frac{\Delta^{2} m_{e} v_{o}^{2}}{15 \lambda_{D}^{2} m_{i} v_{e}^{2}} \omega_{p e}
$$

The result is self consistent only when the pump power is sufficiently large which satisfies

$$
\frac{v_{o}^{2}}{v_{e}^{2}}>15 \cdot\left(\frac{m_{i}}{m_{e}}\right)^{1 / 2} \frac{k \lambda_{D}^{3}}{\Delta^{2}} .
$$

and

$$
\left(\frac{m_{e}}{m_{i}}\right)^{1 / 2} \frac{\Delta}{\lambda_{D}}<1 .
$$

The localization of the instabilities in this case is symmetric about the pump and its width is 


$$
\underset{\therefore}{\therefore} \sim\left|x_{1}-x_{2}\right| \sim 80 \frac{\mathrm{k} \lambda_{\mathrm{D}}^{4}}{\Delta^{3}} \frac{\mathrm{m}}{\mathrm{m}} \frac{\mathrm{v}^{2}}{\mathrm{v}_{\mathrm{o}}^{2}}
$$

We have also tried to find solutions for other values of $\beta$ and $\lambda$. It turns out that either the results are similar to what we have in Eq. (38) to within a numerical factor or else the results are unphysical.

\section{SUMMARY AND DISCUSSTON}

The growth rate and the threshold value for OTSI in the présence of a pump with finite width $\Delta$ have been calculated and summarized in Tablë I. The inhomogeneity of the pump causes a convective loss in addition to the ordinary electron damping of the instability. Thus, the threshold for waves confined within $\Delta$ is lower than that for outside $\Delta$, and both reduce to the threshold for homogeneous pump when $\Delta$ becomes very large.

At low pump power, the instabilities localized within $\Delta$ will grow as $v_{0}^{2}$, while those outside $\Lambda$ will grow as $v_{0}^{8 / 3}$. The localization of the waves outside $\Delta$ is on the overdense side and shifts toward and centers at the plasma frequency as the pump intensity increases. When the pump power is very large, the growth rate $\operatorname{can}^{-1}$ be much greater than $\mathrm{c}_{\mathbf{s}} \mathrm{k}$. If $\left(m_{e} / m_{i}\right)^{1 / 2} \Delta / \lambda_{D}>1$, the waves will have growth rate $\omega_{i} \sim v_{0}^{2 / 3}$ and are confined within $\Delta$. If $\left(m_{e^{/}} / m_{i}\right)^{1 / 2} \Delta / \lambda_{D}<i l$, then the waves wili have turning points outside $\Delta$ and grow as $v_{0}^{2}$. 
The physical nature of the oscillating two-stream instability at the resonance is the following. The linearly transformed plasma wave with nonuniform amplitude produces a ponderomotive force on the electrons: $f_{p}=-e^{2} \nabla\left|E_{p}\right|^{2} / 2 m \omega_{p}^{2}$. The gradient of the intensity is due to, (i) the natural shape of the resonance with width $\Delta$, and (ii) any perturbation of the amplitude with wavelength $2 \pi / k$. This ponderomotive force is stronger, the shorter the wavelength. This ponderomotive force on the fluid is opposed by the thermal pressure of the plasma. The balance of these two forces would therefore produce a density cavity where the field intensity is high. The density 'cavity'in turn would trap more plasmons, leading to the growth of the local field and the deepening of the cavity. Nonlinearly, this growth leads to the formation of solitons in one-dimension where the nonlinearity for spatial condensation is balanced by diffraction, provided the incident e.m. wave is switched off. In two or more dimensions, the soliton collapses spatially and can only be limited by the Landau damping. In an expanding plasma, this ponderomotive force of the localized field near the singular point tends to retard the motion in the overdense region while accelerating it in the underdense region, leading to the formation of a shock with the jump in density near the singular point. This shock formation enhances the linear conversion by transforming into an additional surface wave. It would, of course, modify the nature of the subsequent parametric 
processes. Since we are dealing with the initial stage of the linearly transformed. wave and its parametric instabilities before the density modification takes place, the effect of a nonlinear density profile is not considered in this paper.

\section{ACKNOWLEDGMENTS}

One of the authors (Y.Y.K.) acknowledges helpful discussions with Dr. R. B. White, Dr. R. L. Berger, and Professor F. W. Perkins. I'his work was supported by U.S. Energy Research and Development Administration Contract $E(11-1)-3073$; and also by U.S. Air Force office of Scientific. Research Contract F44620-70-C-0033.

One of the authors (C.S.L.) was, supported by ERDA Contract AT (11-1) -3237 while at the Institute for Advanced Study in Princeton, N.J. 
APPENDIX A

As $|B \Delta| \ll 1$ and $|\lambda \Delta|>1$, the roots for $F(\dot{x})=0$ are

$\left\{\begin{array}{l}\frac{x_{1}}{\Delta} \sim \frac{1}{2 \beta \Delta}\left[4-\frac{1}{2^{1 / 3}}\left(\lambda \beta \Delta^{2}\right)^{2 / 3}-i\left(2 \beta \Delta-\frac{\sqrt{3}}{2^{1 / 3}}\left(\lambda \beta \Delta^{2}\right)^{2 / 3}\right)\right] \\ \frac{x_{2}}{\Delta} \sim \frac{1}{2 \beta \Delta}\left[2^{2 / 3}\left(\lambda \beta \Delta^{2}\right)^{2 / 3}+i\left(\frac{2}{3} \beta \Delta\right)\right] \\ \frac{x_{3}}{\Delta} \sim \frac{1}{2 \beta \Delta}\left[-2^{2 / 3}\left(\lambda \beta \Delta^{2}\right)^{2 / 3}+i\left(\frac{2}{3} \beta \Delta+\frac{1}{2}\left(\lambda \beta \Delta^{2}\right)^{2 / 3}\right)\right] \\ \frac{x_{4}}{\Delta^{*}} \sim \frac{1}{2 \beta \Delta}\left[-2^{1 / 3}\left(\lambda \beta \Delta^{2}\right)^{2 / 3}+i\left(\frac{2}{3} \beta \Delta-2^{1 / 3} \sqrt{3}\left(\lambda \beta \Delta^{2}\right)^{2 / 3}\right)\right]\end{array}\right.$

which yields $\left|\frac{x_{1}}{\Delta}\right|>1$, and $\left|\frac{x_{2}}{\Delta}\right|,\left|\frac{x_{3}}{\Delta}\right|,\left|\frac{x_{4}}{\Delta}\right|>1$. we may. approximate the integral as

$I_{1}=\int_{x_{1}}^{x_{2}}[F(x)]^{1 / 2} d x \sim \beta \int_{x_{1}}^{x_{2}} \frac{\left[\left(x_{1}-x\right)\left(x-x_{2}\right)\left(x-x_{J}\right)\left(x-x_{4}\right)\right]^{1 / 2}}{x^{2}} d x$

$$
\sim-\beta\left(x_{1}-x_{2}\right) B\left(\frac{3}{2}, \frac{1}{2}\right)
$$

where $B$ is Beta function. 
The other integral to be evaluated is

$$
\begin{aligned}
& I_{2}=\int_{x_{2}}^{x_{4}}[F(x)]^{1 / 2} d x \sim \beta \int_{x_{2}}^{x_{4}} 4 \frac{x_{1}^{1 / 2}\left[\left(x-x_{2}\right)\left(x-x_{3}\right)\left(x-x_{4}\right)\right]^{1 / 2}}{x^{2}} d x \\
& \sim \beta \Gamma(2) \Gamma\left(\frac{3}{2}\right) x_{1}^{1 / 2}\left(x_{3}-x_{2}\right)^{1 / 2}\left[\frac{\Gamma\left(-\frac{1}{2}\right)}{\left[1\left(\frac{3}{2}\right)\right]^{2}} F\left(2,-\frac{1}{2}, \frac{3}{2}, \frac{x_{2}}{x_{4}-x_{2}}\right)\right. \\
& \left.-i \frac{\Gamma\left(\frac{1}{2}\right)}{[\Gamma(2)]^{2}}\left(\frac{x_{4}-x_{2}}{x_{2}}\right)^{1 / 2} F\left(\frac{3}{2},-1, \frac{1}{2}, \frac{x_{2}}{x_{4}-x_{2}}\right)\right]
\end{aligned}
$$

where $F$ is Gauss' hypergeometric function. The imaginary part of $I_{2}$ is of the order of $2 \pi\left(\lambda \beta \Delta^{2}\right)^{2 / 3}$ that makes exp $\left\{2 i I_{2}\right\} \sim 0$. Thus, the eigenequation eventually becomes

$$
\int_{x_{1}}^{x_{2}} \because[F(x)]^{1 / 2} \cdot d x=\left(n+\frac{1}{2}\right) \pi
$$

which gives us for the lowest mode

$$
4-\frac{3}{2^{1 / 3}}\left(\lambda \beta \Delta^{2}\right)^{2 / 3}-i \frac{8}{3} \beta \Delta=-2 \text {. }
$$




\section{APPENDIX B}

$$
\begin{aligned}
& \text { As }|\beta \Delta|>>1,|\lambda \Delta|>1 \text { and }\left|\frac{\lambda}{\beta}\right|>1 \text {, the roots for } \\
& F(x)=0 \text { are }
\end{aligned}
$$

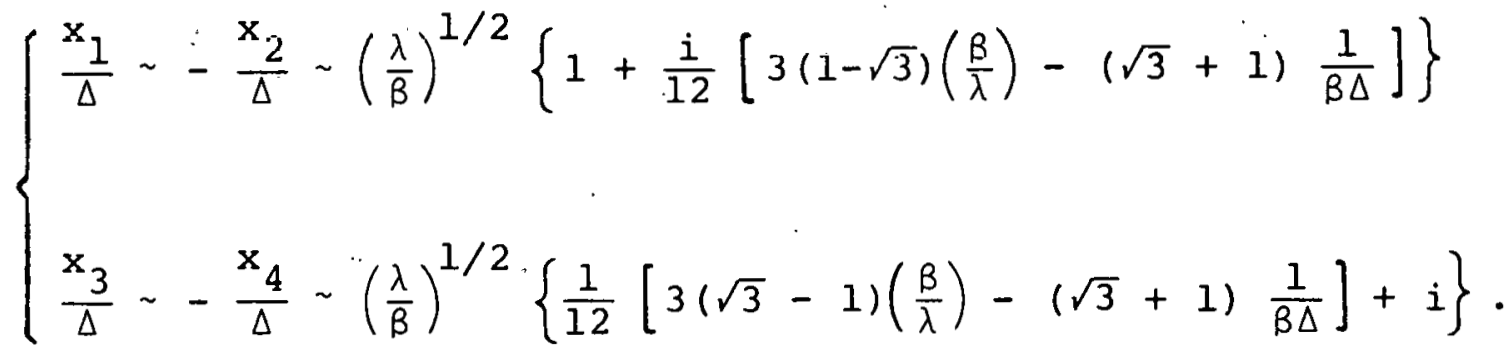

Because $\left|\frac{\lambda^{2} \Delta^{4}}{x_{T}^{4}}\right|>\left|\frac{2 \beta}{x_{T}}\right|$, we may write $F(x) \cong \frac{\lambda^{2}}{\left[\left(\frac{x}{\Delta}\right)^{2}+1\right]^{2}}-\beta^{2}$. Then, as. $\left|\frac{x_{1}}{\Delta}\right| \gg 1$,

$$
\begin{aligned}
I & =\int_{x_{1}}^{x_{2}}[F(x)]^{1 / 2} d x \sim 2 \int_{0}^{x_{2}}[F(x)]^{1 / 2} d x \\
& \sim \sqrt{2} \frac{\lambda \Delta}{x_{1}} B\left(\frac{1}{2}, \frac{3}{2}\right) F_{1}\left(\frac{3}{2},-\frac{1}{2}, 1,2 ; \frac{1}{2}, 1\right) \\
& \sim-\frac{4 \sqrt{2} \pi^{3 / 2} \lambda \Delta^{2}}{\left[\Gamma\left(\frac{1}{4}\right)\right]^{2} x_{3}} .
\end{aligned}
$$


The eigenequation.

$$
\int_{x_{1}}^{x_{2}}[F(x)]^{1 / 2} d x=\left(n+\frac{1}{2}\right) \pi
$$

gives us for the lowest mode:

$$
\left(\lambda: \beta \Delta^{2}\right)^{1 / 2}=\frac{8(2 \pi)^{1 / 2}}{\left[\Gamma\left(\frac{1}{4}\right)\right]^{2}} .
$$




\section{REFERENCES}

$1_{V}$. L. Ginzburg, Propagation of Electromagnetic Waves in.. Plasma, Chapter IV, (Gordon and Breach, New York).

${ }^{2}$ H. C. Kim, R. L. Stenzel, and A. Y. Wong, Phys. Rev. Lett. 33, $896(1974)$; R. L. Stenzel, A. Y. Wong, and H. C. Kim, Phys. -Rev. Lett. 32,654 (1974).

${ }^{3}$ R. B. White, C. S. Liu, and M. N. Rosenbluth, Phys. Rev. Lett. 31,520 (1973).

4D. W. Forslund, J. M. Kindel, K. Lee, and E. L. Lindman, Phys. Rev. Lett. 34, 193 (1975); E. J. Valeo, W. L. Kruer, Phys. Rev. Lett. 33,750 (1974).

${ }^{5}$ K. Nishikawa, J. Phys: Soc. Japan 24, 916 (1968);

K. Nishikawa, J. Phys. Soc. Japan 24, 1152 (1968). 
Table I

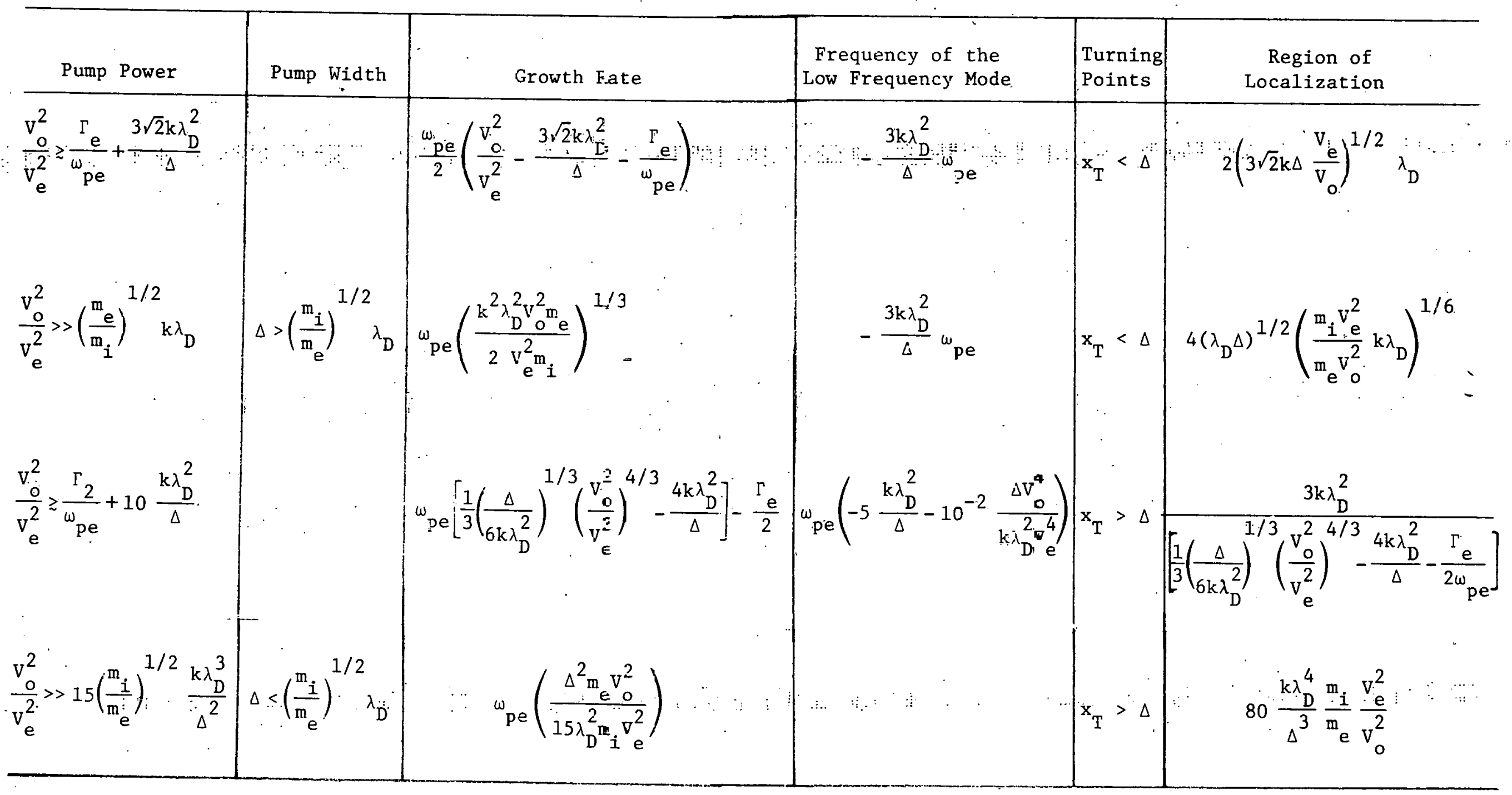




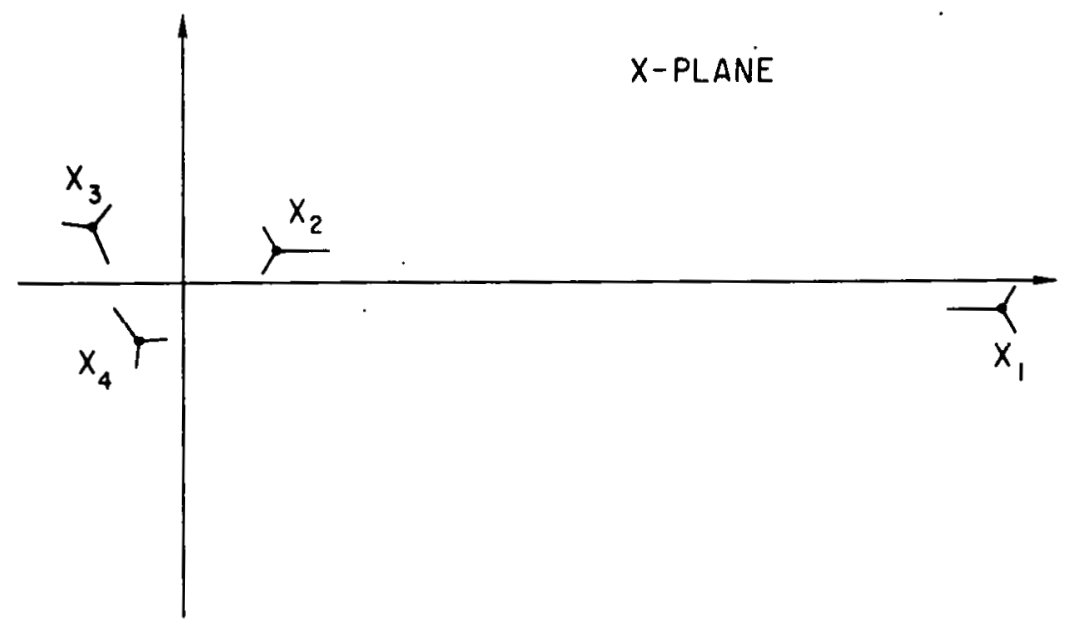

752.195

Fig. 1. The turning points and anti-stokes lines for $|\beta \Delta| \ll 1\left|\frac{x_{1}}{\Delta}\right| \gg 1,\left|\frac{x_{2}}{\Delta}\right| \sim\left|\frac{x_{3}}{\Delta}\right| \sim\left|\frac{x_{4}}{\Delta}\right| \geqslant 1$.

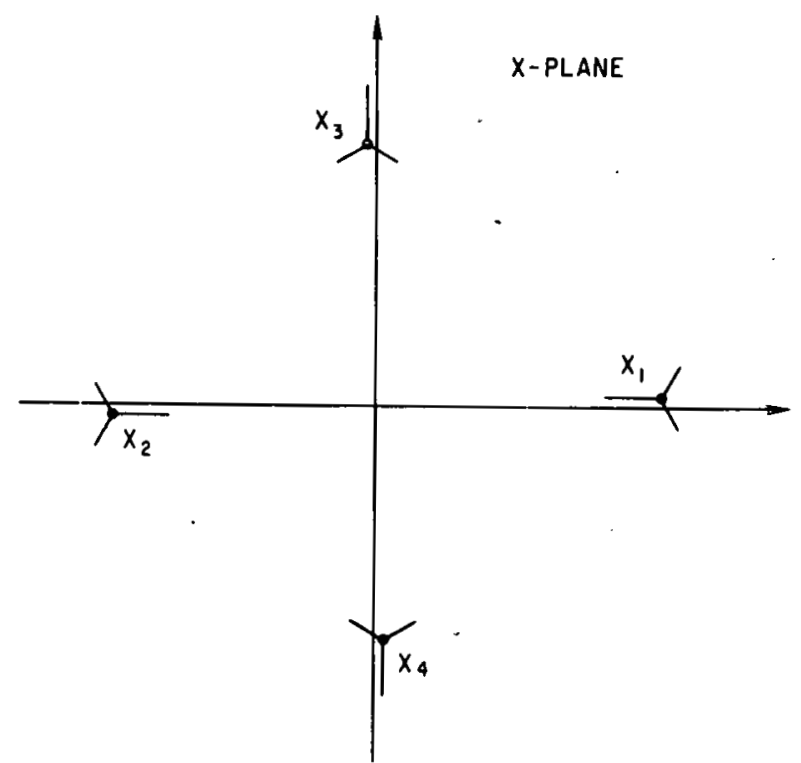

753194

Fig. 2. The turning points and anti-stokes lines for $|\beta \Delta| \gg 1$. 